\title{
Mediterranean Diet Maintained Platelet Count within a Healthy Range and Decreased Thrombocytopenia-Related Mortality Risk: A Randomized Controlled Trial
}

\author{
Álvaro Hernáez ${ }^{1,2,3,4, *}$, Camille Lassale ${ }^{2,5} \oplus$, Sara Castro-Barquero $1,2,6 \oplus$, Emilio Ros ${ }^{1,2,7}$,

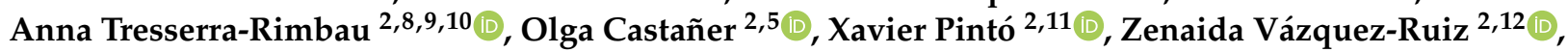 \\ José V. Sorlí ${ }^{2,13}{ }^{\circledR}$, Jordi Salas-Salvadó ${ }^{2,9,10}{ }^{\circledR}$, José Lapetra ${ }^{2,14}$, Enrique Gómez-Gracia ${ }^{15}$, \\ Ángel M. Alonso-Gómez ${ }^{2,16} \mathbb{D}$, Miquel Fiol 2,17, Lluis Serra-Majem ${ }^{2,18,19}$, Emilio Sacanella 1,2,6,20®,

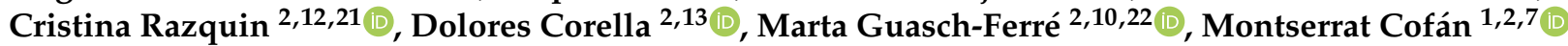 \\ and Ramón Estruch 1,2,6,20
}

\section{check for}

updates

Citation: Hernáez, Á.; Lassale, C.; Castro-Barquero, S.; Ros, E.; TresserraRimbau, A.; Castañer, O.; Pintó, X.; Vázquez-Ruiz, Z.; Sorlí, J.V.; SalasSalvadó, J.; et al. Mediterranean Diet Maintained Platelet Count within a Healthy Range and Decreased Thrombocytopenia-Related Mortality Risk: A Randomized Controlled Trial Nutrients 2021, 13, 559. https:// doi.org/10.3390/nu13020559

Academic Editor: Martina Barchitta

Received: 8 January 2021

Accepted: 3 February 2021

Published: 8 February 202

Publisher's Note: MDPI stays neutral with regard to jurisdictional claims in published maps and institutional affiliations.

Copyright: (c) 2021 by the authors. Licensee MDPI, Basel, Switzerland. This article is an open access article distributed under the terms and conditions of the Creative Commons Attribution (CC BY) license (https:/ / creativecommons.org/licenses/by/ $4.0 /)$
1 August Pi i Sunyer Biomedical Research Institute (IDIBAPS), 08036 Barcelona, Spain; sacastro@clinic.cat (S.C.-B.); eros@clinic.cat (E.R.); esacane@clinic.cat (E.S.); MCOFAN@clinic.cat (M.C.); restruch@clinic.cat (R.E.)

2 Consorcio CIBER, M.P. Fisiopatología de la Obesidad y Nutrición (CIBEROBN), Instituto de Salud Carlos III, 28029 Madrid, Spain; classale@imim.es (C.L.); annatresserra@ub.edu (A.T.-R.); ocastaner@imim.es (O.C.); xpinto@bellvitgehospital.cat (X.P.); zvazquez@unav.es (Z.V.-R.); jose.sorli@uv.es (J.V.S.); jordi.salas@urv.cat (J.S.-S.); joselapetra543@gmail.com (J.L.); angelmago13@gmail.com (Á.M.A.-G.); miguel.fiol@ssib.es (M.F.); lserra@dcc.ulpgc.es (L.S.-M.); crazquin@unav.es (C.R.); dolores.corella@uv.es (D.C.); mguasch@hsph.harvard.edu (M.G.-F.)

3 Blanquerna School of Health Sciences, Universitat Ramon Llull, 08025 Barcelona, Spain

4 Centre for Fertility and Health (CeFH), Norwegian Institute of Public Health, 0473 Oslo, Norway

5 Cardiovascular Risk and Nutrition Research Group, Hospital del Mar Medical Research Institute (IMIM), 08003 Barcelona, Spain

6 Department of Medicine, Faculty of Medicine and Health Sciences, University of Barcelona, 08036 Barcelona, Spain

7 Lipid Clinic, Endocrinology and Nutrition Service, Hospital Clínic, 08036 Barcelona, Spain

8 Department of Nutrition, Food Science and Gastronomy, XaRTA, INSA, Faculty of Pharmacy and Food Sciences, University of Barcelona, 08028 Barcelona, Spain

9 Unitat de Nutrició Humana, Departament de Bioquimica i Biotecnologia, Universitat Rovira i Virgili, Hospital Universitari Sant Joan de Reus, 43201 Reus, Spain

10 Institut d'Investigació Pere Virgili (IISPV), 43204 Reus, Spain

11 Lipids and Vascular Risk Unit, Internal Medicine Service, Hospital Universitario de Bellvitge, 08907 L'Hospitalet de Llobregat, Spain

12 Department of Preventive Medicine and Public Health, Universidad de Navarra, 31008 Pamplona, Spain

13 Department of Preventive Medicine, Universidad de Valencia, 46010 Valencia, Spain

14 Research Unit, Department of Family Medicine, Distrito Sanitario Atención Primaria Sevilla, 41013 Sevilla, Spain

15 Department of Preventive Medicine and Public Health, Universidad de Málaga, 29071 Málaga, Spain; egomezgracia@uma.es

16 Bioaraba Health Research Institute, Osakidetza Basque Health Service, Araba University Hospital, University of the Basque Country UPV/EHU, 01009 Vitoria-Gasteiz, Spain

17 Health Research Institute of the Balearic Islands (IdISBa), Hospital Son Espases, 07120 Palma de Mallorca, Spain

18 Instituto de Investigaciones Biomédicas y Sanitarias, Universidad de Las Palmas de Gran Canaria, 35016 Las Palmas, Spain

19 Centro Hospitalario Universitario Insular Materno Infantil (CHUIMI), Servicio Canario de Salud, 35016 Las Palmas, Spain

20 Internal Medicine Service, Hospital Clínic, 08036 Barcelona, Spain

21 Department of Endocrinology and Nutrition, Complejo Hospitalario de Navarra, 31008 Pamplona, Spain

22 Department of Nutrition, Harvard TH Chan School of Public Health, Boston, MA 02115, USA

* Correspondence: alvaro.hernaez1@gmail.com; Tel.: +34-679384179

Abstract: There is little information on the dietary modulation of thrombosis-related risk factors such as platelet count. We aimed to assess the effects of Mediterranean diet (MedDiet) on platelet count and related outcomes in an older population at high cardiovascular risk. In participants of the 
PREDIMED (PREvención con DIeta MEDiterránea) study, we assessed whether an intervention with a MedDiet enriched with extra-virgin olive oil or nuts, relative to a low-fat control diet, modulated platelet count $(n=4189)$, the risk of developing thrombocytosis and thrombocytopenia $(n=3086)$, and the association between these alterations and all-cause mortality (median follow-up time: 3.0 years). Although platelet count increased over time $\left(+0.98 \cdot 10^{9}\right.$ units/L.year [95\% confidence interval: 0.12 ; 1.84]), MedDiet interventions moderated this increase, particularly in individuals with near-high baseline count (both MedDiets combined: $-3.20 \cdot 10^{9}$ units/L·year $[-5.81 ;-0.59]$ ). Thrombocytopenia incidence was lower in the MedDiet interventions (incidence rates: $2.23 \%$ in control diet, $0.91 \%$ in MedDiets combined; hazard ratio: 0.44 [0.23; 0.83]). Finally, thrombocytopenia was associated with a higher risk of all-cause mortality (hazard ratio: 4.71 [2.69; 8.24]), but this relationship was attenuated in those allocated to MedDiet ( $p$-interaction $=0.018$ ). In brief, MedDiet maintained platelet counts within a healthy range and attenuated platelet-related mortality in older adults at high cardiovascular risk.

Keywords: Mediterranean diet; platelet count; thrombocytopenia; randomized controlled trial; prevention

\section{Introduction}

Accruing evidence from observational studies and randomized controlled trials such as the PREDIMED (PREvención con DIeta MEDiterránea) study indicates that following a Mediterranean diet (MedDiet) reduces the risk of developing cardiovascular outcomes [1,2]. It is believed that MedDiet may exert these benefits via improving glucose and lipid metabolism, enhancing endothelial function, and decreasing oxidative stress and low-grade inflammation [3]. However, little is known about the effects of MedDiet on thrombosis-related indicators. Following a MedDiet has been associated with improvements in atherothrombosis biomarkers [4], platelet function [5], and decreases in the circulating levels of prothrombotic microvesicles [6]. However, there is little information on the effects of this dietary pattern on other markers related to thrombosis of a more clinical nature, such as platelet count. Platelets are key physiological initiators in clot formation but are increasingly being associated with pathophysiological mechanisms of disease independent of thrombosis $[7,8]$. Defective and excessive platelet count has been associated with increased mortality and cardiovascular disease incidence in the general population and high cardiovascular risk individuals [9-11]. Therefore, finding preventive strategies to avoid the appearance of these alterations seems essential. Following another plant-based dietary pattern (vegan diet) for 4 weeks decreased platelet counts in a small population relative to a meat-rich eating plan $(n=53)$ [12]. High adherence to a MedDiet has only been associated with decreased platelet count in a cross-sectional study [13] and in a small-scale, short-term intervention trial [14]. However, no study has assessed the effects of this dietary pattern on thrombocytosis in the long term and in larger populations, or on other platelet count-related alterations, such as thrombocytopenia, likely to appear in individuals with poor nutritional status [15]. We also do not know whether a possible improvement in platelet count could partially mediate the beneficial effects of MedDiet on chronic diseases and their associated mortality [16].

The aims of our study were to assess in older individuals at high cardiovascular risk whether an intervention with MedDiet (1) maintained platelet count within a healthy range and decreased the risk of developing platelet count-related disorders (thrombocytosis and thrombocytopenia); and (2) modified the association between platelet count-related disorders and all-cause mortality.

\section{Materials and Methods}

\subsection{Study Population}

The study population consisted of participants of the PREDIMED study, which was a multicenter, randomized, controlled trial conducted in Spain between 2003 and 2010 assessing the effects of following a MedDiet on the primary prevention of cardiovascular 
outcomes in an older population at high cardiovascular risk. Eligible participants were men (aged 55-80 years) and women (aged 60-80 years) free of cardiovascular disease at enrolment but with type-2 diabetes or three or more of the following risk factors: (1) smoking; (2) hypertension; (3) high concentrations of low-density lipoprotein cholesterol; (4) low levels of high-density lipoprotein cholesterol; (5) overweight/obesity; and (6) family history of premature coronary heart disease. Enrolment commenced on 25 June 2003, and the last participant was included on 30 June 2009. The PREDIMED study was registered under the International Standard Randomized Controlled Trial Number ISRCTN35739639 (http: / / www.isrctn.com/ISRCTN35739639 (accessed on 8 January 2021)). The study protocol complied with the Declaration of Helsinki and was endorsed by institutional review boards of all recruiting centers. An institutional ethics committee (CEIC-PSMAR) approved the particular protocol of this sub-project (code: 2018/8180/I; date: 4 December 2018). All volunteers provided written informed consent before entering the trial. The protocol, recruiting methods, and data collection processes have been described in previous publications $[1,17]$ and are available in the PREDIMED study website (http: / / www.predimed.es (accessed on 8 January 2021)).

Platelet count alterations were not a predefined endpoint in the PREDIMED study, and thus this study should be considered as exploratory. Of the 4381 PREDIMED participants with blood count available, we excluded 28 individuals without baseline data on MedDiet adherence or intake of alcohol, folate, and iron. To exclusively determine the effects on platelet levels of the dietary intervention, we also excluded those participants with any condition potentially related to alterations in platelet count at any point of the study $[15,18]$, including the following: (1) any cancer of the immune system (17 individuals); (2) an autoimmune disease ( 28 individuals, determined as the use of immunosuppressant medications); (3) alcohol abuse (26 individuals, determined as a cumulative average of alcohol intake throughout the study of $\geq 4$ drinks / day in men or $\geq 3$ drinks / day in women); (4) viral infections (9 participants, determined as the use of oral antiviral medication); and (5) users of medications associated with platelet count alterations (10 users of heparins, 31 users of certain anticonvulsants (carbamazepine, phenytoin, and valproate), 32 users of L-dopa, 8 users of certain antibiotics (sulfamethoxazole, sulfa antibiotics, and vancomycin), and 3 fluconazole users). No participants with health outcomes related to altered platelet count (aplastic anemia, myelodysplastic syndromes, paroxysmal nocturnal hemoglobinuria, thrombotic thrombocytopenic purpura, disseminated intravascular coagulation syndrome, hemolytic uremic syndrome, Wiskott-Aldrich syndrome, May-Hegglin anomaly, etc.) were included in the trial. This yielded a main analytical sample of 4189 participants. For the analyses on the risk of developing platelet count-related alterations, we additionally excluded those individuals with thrombocytopenia or thrombocytosis at baseline (81 and 111, respectively) and those without information of platelet count in follow-up visits (911 participants) (sample size: 3086 individuals). The flowchart of the study is available in Figure 1.

\subsection{Dietary Intervention}

Three intervention arms (to which volunteers were randomly allocated according to a 1:1:1 ratio) were compared: (1) a MedDiet enriched with extra-virgin olive oil (MedDietEVOO); (2) a MedDiet enriched with mixed nuts (MedDiet-Nuts); and (3) a low-fat control diet. MedDiet interventions encouraged (1) the intake of vegetables, fruits, pulses, nuts, and fish; (2) the use of extra-virgin olive oil as the main culinary fat; (3) a reduction in the consumption of sugary drinks, commercial bakery goods, pastries, sweets, and spread fats; (4) the replacement of red/processed meats for poultry; and (5) food preparation following home-made recipes (such as the traditional "sofrito", tomato-based stir-fried sauce with olive oil, onion, and garlic). To promote compliance and account for family needs, volunteers allocated in the MedDiet-EVOO intervention received $1 \mathrm{~L} /$ week of extravirgin olive oil and those in the MedDiet-Nuts group were provided with $210 \mathrm{~g} /$ week of mixed nuts. Volunteers allocated to the low-fat control group were advised (1) to increase 
the consumption of vegetables, fruits, pulses, low-fat dairy products, and lean fish; and (2) to reduce their intake of vegetable oils (including olive oil), commercial bakery goods and sweets, nuts and fried snacks, fatty fish, seafood canned in oil, red and processed fatty meats, visible fat in meats and soups, spread fats, and "sofrito". Additional details of the dietary protocol are available elsewhere $[1,17]$.

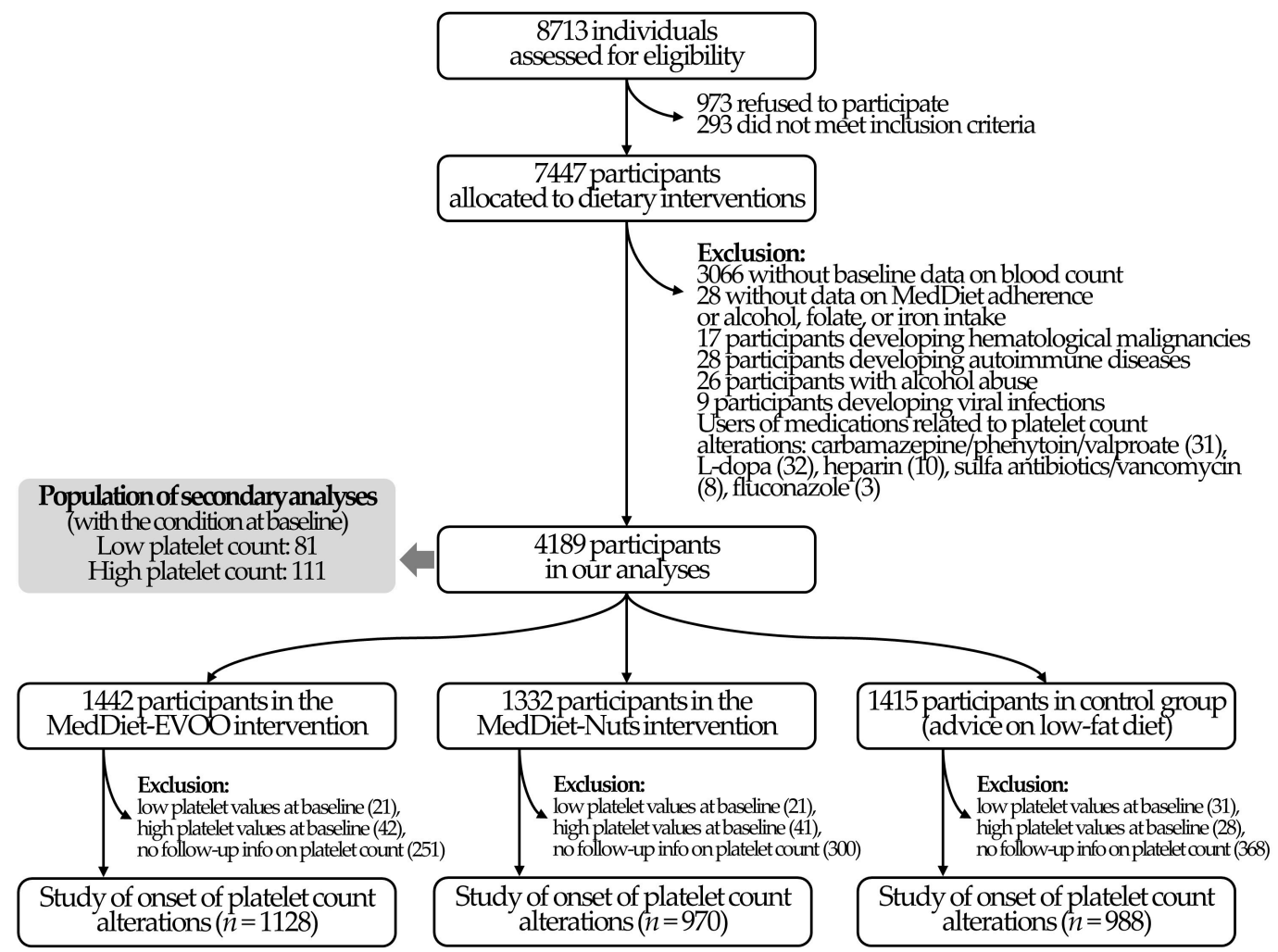

Figure 1. Study flowchart. MedDiet: Mediterranean diet; MedDiet-EVOO: MedDiet enriched with extra-virgin olive oil; MedDiet-Nuts: MedDiet enriched with mixed nuts.

\subsection{Outcomes}

Platelet count was measured at baseline in all 4189 participants in the main analytical sample and at least in one study follow-up point in 3086 individuals in fasting blood samples by automated analyzers [19]. The normal range in an older Mediterranean population is 122 to $350 \times 10^{9}$ platelets/L in men and 140 to $379 \times 10^{9}$ platelets/L in women [20]. Thus, thrombocytopenia (low platelet count) was defined as presenting $<122$ in men and $<140 \cdot 10^{9}$ units/L in women, and thrombocytosis (high platelet count) was defined as presenting $>350$ in men and $>379 \cdot 10^{9}$ units/L in women. We calculated incidence and time-to-event of the onset of any of these alterations among volunteers with platelet counts within the physiological range at study baseline. We defined "onset" as the occurrence of any of the platelet count alterations in one of the follow-up visits that persisted until the last visit for which data is available. We considered a valid onset as any occurrence that persisted for at least three subsequent follow-up visits and presented no more than one "return to normal" value.

For our second aim, we collected information on all-cause mortality. Occurrence up to 1 December 2010 and time-to-event values were determined by the Clinical Event Committee through follow-up study visits, yearly review of medical records, repeated contact with the participants, and linkage with the national death registry [1,17]. 


\subsection{Covariates}

Trained personnel collected data on the following variables in the baseline visit: age; sex; educational level; prevalence of diabetes, hypercholesterolemia, hypertriglyceridemia, and hypertension; use of antiplatelet drugs; body mass index; and smoking habit. To account for any indicators related to low-grade inflammation, white blood cell counts were measured in fasting blood samples in automated analyzers, as previously described [19]. We estimated physical activity levels in metabolic equivalents of task-minute per day from the Minnesota Leisure-Time Physical Activity Questionnaire validated in the Spanish population. Finally, we estimated the intake of alcohol (in g/day), dietary folate (in $\mu \mathrm{g} /$ day), and iron (in $\mathrm{mg} /$ day) from a validated 137-item food frequency questionnaire [1,17].

\subsection{Power Analyses}

The number of total individuals and cases that occurred during follow-up in each study group allowed $\geq 80 \%$ power to detect as significant $(p$-value $<0.05$ ) hazard ratios (HR) lower than 0.42, 0.35, and 0.33 (for the comparisons of both MedDiets combined, MedDiet-EVOO, and MedDiet-Nuts, with control diet, respectively) in relation to the onset of thrombocytopenia, and lower than $0.48,0.40$, and 0.35 regarding the onset of thrombocytosis (Supplementary Materials Table S1). We performed these analyses using the "powerSurvEpi" package in R Software [21].

\subsection{Statistical Analyses}

We described the characteristics of the participants at baseline by means and standard deviation (normally distributed continuous variables), medians and interquartile range (non-normally distributed continuous variables), and proportions (categorical variables).

We studied the effects of MedDiet interventions on platelet count evolution by repeated measurement mixed linear models. We assessed time effects (continuous yearly change across the overall study population) and between-group changes (difference in changes over time in the MedDiet intervention arms—individually and combined-relative to the control diet group). We performed the previous analyses in the overall study population, as well as stratifying participants in quartiles according to platelet count at baseline (after testing whether there was a significant interaction between baseline platelet count and intervention group, by applying a likelihood ratio test between the nested models with and without the interaction product term). Estimates were adjusted for age (continuous), sex, educational level (primary, secondary, greater, unavailable information), recruitment site, diabetes (yes/no), hypercholesterolemia (yes/no), hypertriglyceridemia (yes/no), hypertension (yes/no), use of antiplatelet drugs (yes/no), smoking habit (current/former/never smoker), white blood cell count (continuous), leisure-time physical activity (continuous), body mass index (continuous), alcohol consumption (continuous), folate intake (continuous), iron consumption (continuous), and two propensity scores that used 30 baseline variables to estimate the probability of assignment to each of the intervention groups [1]. We fitted models using the "lme4" package in R Software (Vienna, Austria) [22].

We assessed the differences in the risk of developing thrombocytopenia and thrombocytosis using two sets of Cox proportional hazards regression models. We defined follow-up time as the time between the date of enrolment and (1) the midpoint between the last visit without the outcome and the first visit in which the alteration was registered [23]; (2) 5 years of maximum follow-up time; or (3) 1 December 2010, whichever came first. Any onset of platelet count alteration after a diagnosis of cancer was not considered a valid outcome ( 3 thrombocytopenias, 3 thrombocytosis). We evaluated whether there were differences in the risk of presenting the outcome in the MedDiet intervention groups (individually and combined) relative to the control diet and fitted two models. Model 1 was stratified by sex and recruitment site and adjusted for age. Model 2 was further stratified by educational level and adjusted for baseline platelet count and the rest of the covariates described in the repeated measurement analyses. We used robust variance estimators to 
minimize intra-cluster correlations [1] and fitted models using the "survival" package in $\mathrm{R}$ Software [24]. We also represented Kaplan-Meier cumulative incidence curves for each study group, weighted by inverse probability weighting with a propensity score model of assignment to intervention or control group based on the covariates above listed.

Finally, we determined whether MedDiet modified the association between platelet count alterations at baseline and all-cause mortality. We compared the volunteers allocated to MedDiet intervention relative to those in the control group. We fitted Cox models where the outcome was major adverse cardiovascular events or all-cause mortality, included an interaction product term of "platelet count alteration $\times$ intervention group", and applied a likelihood ratio test between the nested models with and without it.

We performed all analyses with R Software, version 3.5.2 [25].

\section{Results}

\subsection{Study Population}

Our participants were older adults (67 years old on average, 58\% women) with a high prevalence of cardiovascular risk factors ( $84 \%$ hypertension, $74 \%$ hypercholesterolemia, $49 \%$ obesity, $47 \%$ diabetes, $30 \%$ hypertriglyceridemia, $13 \%$ current smokers) (Table 1 ), followed for a median time of 3.0 years.

Table 1. Study population ${ }^{1}$.

\begin{tabular}{|c|c|c|c|c|}
\hline & $\begin{array}{l}\text { All Participants } \\
\quad(n=4189)\end{array}$ & $\begin{array}{l}\text { MedDiet-EVOO } \\
\quad(n=1442)\end{array}$ & $\begin{array}{l}\text { MedDiet-Nuts } \\
\quad(n=1332)\end{array}$ & $\begin{array}{c}\text { Control Diet } \\
\quad(n=1415)\end{array}$ \\
\hline Age (years), mean $\pm S D$ & $67.0 \pm 6.14$ & $66.7 \pm 6.06$ & $66.9 \pm 6.04$ & $67.5 \pm 6.30$ \\
\hline Female sex, $n(\%)$ & $2430(58.0)$ & $859(59.6)$ & $725(54.4)$ & $846(59.8)$ \\
\hline Diabetes, $n(\%)$ & $1954(46.6)$ & $686(47.6)$ & $611(45.9)$ & $657(46.4)$ \\
\hline Hypercholesterolemia, $n(\%)$ & $3100(74.0)$ & $1069(74.1)$ & $996(74.8)$ & $1035(73.1)$ \\
\hline Hypertriglyceridemia, $n$ (\%) & $1264(30.2)$ & $439(30.4)$ & $405(30.4)$ & $420(29.7)$ \\
\hline Hypertension, $n(\%)$ & $3522(84.1)$ & $1201(83.3)$ & $1121(84.2)$ & $1200(84.8)$ \\
\hline Antiplatelet users, $n(\%)$ & $830(19.8)$ & 255 (17.7) & $284(21.3)$ & $291(20.6)$ \\
\hline \multicolumn{5}{|l|}{ Smoking habit: } \\
\hline Never smokers, $n(\%)$ & $2589(61.8)$ & $895(62.1)$ & $798(59.9)$ & $896(63.3)$ \\
\hline Current smokers, $n(\%)$ & $562(13.4)$ & $195(13.5)$ & $186(14.0)$ & $181(12.8)$ \\
\hline Former smokers, $n(\%)$ & $1038(24.8)$ & $352(24.4)$ & $348(26.1)$ & $338(23.9)$ \\
\hline \multicolumn{5}{|l|}{ Weight (according to body mass index): } \\
\hline $18.5-24.9 \mathrm{~kg} / \mathrm{m}^{2}, n(\%)$ & $281(6.71)$ & $100(6.93)$ & $101(7.58)$ & $80(5.65)$ \\
\hline $25.0-29.9 \mathrm{~kg} / \mathrm{m}^{2}, n(\%)$ & $1860(44.4)$ & $645(44.7)$ & $610(45.8)$ & $605(42.8)$ \\
\hline$\geq 30.0 \mathrm{~kg} / \mathrm{m}^{2}, n(\%)$ & $2048(48.9)$ & $697(48.3)$ & $621(46.6)$ & $730(51.6)$ \\
\hline $\begin{array}{l}\text { Leisure-time physical activity } \\
\text { (metabolic equivalents of task-min/day), } \\
\text { median (1st-3rd quartile) }\end{array}$ & $166(56.1-312)$ & $175(60.8-319)$ & $182(63.3-326)$ & $150(46.4-280)$ \\
\hline
\end{tabular}

${ }^{1}$ MedDiet-EVOO: Mediterranean diet intervention enriched with extra-virgin olive oil; MedDiet-Nuts: Mediterranean diet intervention enriched with mixed nuts.

\subsection{MedDiet Interventions and Platelet Count}

Overall platelet count increased over the follow-up in the whole study population $\left(+0.98 \cdot 10^{9}\right.$ units / L year [95\% confidence interval: $\left.\left.0.12 ; 1.84\right]\right)$. However, the MedDiet-Nuts intervention moderated this increase throughout the study $\left(-1.21 \cdot 10^{9}\right.$ units $/ \mathrm{L} \cdot$ year $[-2.39$; -0.027], less increase than the control low-fat group). Intervention effects depended on baseline platelet levels ( $p$-value for interaction with both MedDiets combined $=0.020$; $p$-value for interaction with MedDiet-EVOO $=0.080$; $p$-value for with MedDiet-Nuts $=0.019$ ). 
In stratified analysis by platelet count at baseline, both MedDiet interventions combined (compared to the low-fat control group) particularly contributed to maintaining the platelet count within a normal range in subjects with near-high baseline counts, namely the fourth quartile $\left(-3.20 \cdot 10^{9}\right.$ units/L·year $\left.[-5.81 ;-0.59]\right)$. The MedDiet-Nuts intervention induced this effect in subjects with baseline levels in the third $\left(-2.17 \cdot 10^{9}\right.$ units/L.year $[-4.13$; $-0.21])$ and fourth quartile $\left(-4.13 \cdot 10^{9}\right.$ units /L.year $\left.[-7.17 ;-1.09]\right)$, whilst a similar outcome was also suggested in the MedDiet-EVOO group in individuals in the fourth quartile $\left(-2.48 \cdot 10^{9}\right.$ units/L·year $\left.[-5.36 ; 0.40]\right)$ (Table 2).

Table 2. Time-dependent changes in platelet count in the PREDIMED study groups ${ }^{1}$.

\begin{tabular}{|c|c|c|c|c|c|c|}
\hline & $\begin{array}{c}\text { Baseline } \\
\text { Mean } \pm S D\end{array}$ & $\begin{array}{c}1 \text { Year } \\
\text { Mean } \pm S D\end{array}$ & $\begin{array}{c}\text { 2-3 Years } \\
\text { Mean } \pm S D\end{array}$ & $\begin{array}{c}4-5 \text { Years } \\
\text { Mean } \pm S D\end{array}$ & $\begin{array}{c}\text { Time Effect } \\
\left(10^{9} \text { Units/L·Year, }\right. \\
[95 \% \text { CI }])\end{array}$ & $\begin{array}{l}\text { Time } \times \text { Group Effect } \\
\left(10^{9} \text { Units } / \text { L } \cdot \text { Year, }[95 \% \text { CI], }\right. \\
\text { vs. Control Diet) }\end{array}$ \\
\hline \multicolumn{7}{|c|}{ All individuals } \\
\hline Control diet & $235 \pm 60.4$ & $236 \pm 60.7$ & $237 \pm 64.1$ & $238 \pm 64.2$ & \multirow{4}{*}{$\begin{array}{l}+0.98 \\
{[0.12 ; 1.84]}\end{array}$} & Ref. \\
\hline MedDiet combined & $235 \pm 60.5$ & $237 \pm 61.9$ & $236 \pm 60.8$ & $236 \pm 65.6$ & & $-0.82[-1.83 ; 0.19]$ \\
\hline MedDiet-EVOO & $236 \pm 60.1$ & $236 \pm 62.7$ & $239 \pm 61.9$ & $239 \pm 67.7$ & & $-0.52[-1.64 ; 0.60]$ \\
\hline MedDiet-Nuts & $235 \pm 61.1$ & $237 \pm 61.1$ & $234 \pm 59.2$ & $232 \pm 62.4$ & & $-1.21[-2.39 ;-0.027]$ \\
\hline \multicolumn{7}{|c|}{ 1st quartile $\left(<194 \cdot 10^{9}\right.$ platelets $/ \mathrm{L}$ at baseline $)$} \\
\hline Control diet & $166 \pm 24.0$ & $180 \pm 40.3$ & $176 \pm 42.0$ & $185 \pm 42.6$ & \multirow{4}{*}{$\begin{array}{l}+3.67 \\
{[2.30 ; 5.04]}\end{array}$} & Ref. \\
\hline MedDiet combined & $168 \pm 23.8$ & $180 \pm 41.8$ & $180 \pm 36.6$ & $183 \pm 43.4$ & & $-0.024[-1.65 ; 1.60]$ \\
\hline MedDiet-EVOO & $167 \pm 24.2$ & $180 \pm 37.9$ & $181 \pm 34.2$ & $182 \pm 41.6$ & & $-0.056[-1.87 ; 1.76]$ \\
\hline MedDiet-Nuts & $168 \pm 23.5$ & $180 \pm 46.2$ & $180 \pm 39.4$ & $184 \pm 45.9$ & & $0.032[-1.87 ; 1.93]$ \\
\hline \multicolumn{7}{|c|}{ 2nd quartile (194-229.109 platelets / L at baseline) } \\
\hline Control diet & $212 \pm 9.71$ & $217 \pm 31.1$ & $217 \pm 34.0$ & $221 \pm 35.0$ & \multirow{4}{*}{$\begin{array}{l}+1.41 \\
{[0.12 ; 2.70]}\end{array}$} & Ref. \\
\hline MedDiet combined & $212 \pm 9.96$ & $219 \pm 36.1$ & $216 \pm 39.4$ & $218 \pm 38.8$ & & $-0.33[-1.84 ; 1.18]$ \\
\hline MedDiet-EVOO & $213 \pm 9.65$ & $218 \pm 35.0$ & $218 \pm 43.6$ & $218 \pm 39.4$ & & $-0.42[-2.10 ; 1.26]$ \\
\hline MedDiet-Nuts & $211 \pm 10.3$ & $220 \pm 37.3$ & $214 \pm 32.4$ & $218 \pm 38.2$ & & $-0.22[-1.99 ; 1.55]$ \\
\hline \multicolumn{7}{|c|}{ 3rd quartile (229-268·109 platelets / L at baseline) } \\
\hline Control diet & $248 \pm 10.9$ & $248 \pm 33.3$ & $245 \pm 34.6$ & $252 \pm 40.3$ & \multirow{4}{*}{$\begin{array}{l}+0.86 \\
{[-0.56 ; 2.28]}\end{array}$} & Ref. \\
\hline MedDiet combined & $248 \pm 11.0$ & $247 \pm 33.2$ & $246 \pm 34.6$ & $252 \pm 48.9$ & & $-0.43[-2.10 ; 1.24]$ \\
\hline MedDiet-EVOO & $249 \pm 11.1$ & $247 \pm 32.3$ & $250 \pm 35.3$ & $256 \pm 43.7$ & & $0.85[-1.00 ; 2.70]$ \\
\hline MedDiet-Nuts & $248 \pm 10.9$ & $247 \pm 34.1$ & $241 \pm 33.3$ & $246 \pm 54.7$ & & $-2.17[-4.13 ;-0.21]$ \\
\hline \multicolumn{7}{|c|}{ 4th quartile $\left(\geq 268 \cdot 10^{9}\right.$ platelets $/ \mathrm{L}$ at baseline $)$} \\
\hline Control diet & $315 \pm 44.5$ & $300 \pm 54.9$ & $305 \pm 56.6$ & $318 \pm 59.4$ & \multirow{4}{*}{$\begin{array}{l}-1.46 \\
{[-3.70 ; 0.78]}\end{array}$} & Ref. \\
\hline MedDiet combined & $316 \pm 45.3$ & $301 \pm 60.7$ & $297 \pm 57.1$ & $299 \pm 67.7$ & & $-3.20[-5.81 ;-0.59]$ \\
\hline MedDiet-EVOO & $316 \pm 43.2$ & $303 \pm 65.5$ & $299 \pm 59.2$ & $306 \pm 70.9$ & & $-2.48[-5.36 ; 0.40]$ \\
\hline MedDiet-Nuts & $317 \pm 47.6$ & $299 \pm 55.2$ & $294 \pm 54.3$ & $288 \pm 61.6$ & & $-4.13[-7.17 ;-1.09]$ \\
\hline
\end{tabular}

1 Time effects and between-group changes were estimated by repeated measurement mixed models adjusted for age, sex, educational level, recruitment site, diabetes, hypercholesterolemia, hypertriglyceridemia, hypertension, antiplatelet drug use, smoking habit, white blood cells, leisure-time physical activity, body mass index, intake of alcohol, folates, and iron (at baseline, all), as well as two propensity scores that used 30 baseline variables to estimate the probability of assignment to each of the intervention groups. MedDiet-EVOO: Mediterranean diet intervention enriched with extra-virgin olive oil; MedDiet-Nuts: Mediterranean diet intervention enriched with mixed nuts.

\subsection{MedDiet Interventions and Platelet Count-Related Disorders}

As observed in Table 3, both MedDiet interventions were associated with a decreased risk of developing thrombocytopenia. Incidence rates were $2.23 \%$ in the control group, $0.91 \%$ in both MedDiets combined, $0.89 \%$ in the MedDiet-EVOO, and $0.93 \%$ in the MedDietNuts. Both MedDiet interventions together decreased the risk of thrombocytopenia by $56 \%$ (HR: 0.44 [0.23; 0.83]) compared to the control group. When analyzing MedDiet arms individually, the MedDiet-EVOO was also associated with less risk (HR: 0.36 [0.16; 0.80]), 
whilst the MedDiet-Nuts was linked to lower risk in the model only adjusted for age, sex, and recruitment site (HR: 0.40 [0.18; 0.89]). There was no evidence of an effect on the risk of developing thrombocytosis. Weighted Kaplan-Meier curves are available in Supplementary Materials Figure S1.

Table 3. Incidence of platelet count-related alterations in the study population ${ }^{1}$.

\begin{tabular}{|c|c|c|c|c|c|c|}
\hline & \multicolumn{3}{|c|}{ Thrombocytopenia } & \multicolumn{3}{|c|}{ Thrombocytosis } \\
\hline & $\begin{array}{c}\text { Cases/Total } \\
\text { (Incidence Rate) }\end{array}$ & $\begin{array}{c}\text { Model } 1 \\
\text { HR [95\% CI] }\end{array}$ & $\begin{array}{c}\text { Model } 2 \\
\text { HR [95\% CI] }\end{array}$ & $\begin{array}{c}\text { Cases/Total } \\
\text { (Incidence Rate) }\end{array}$ & $\begin{array}{c}\text { Model } 1 \\
\text { HR [95\% CI] }\end{array}$ & $\begin{array}{c}\text { Model } 2 \\
\text { HR [95\% CI] }\end{array}$ \\
\hline Control diet & $\begin{array}{l}22 / 988 \\
(2.23 \%)\end{array}$ & 1 (Ref.) & 1 (Ref.) & $\begin{array}{l}18 / 988 \\
(1.82 \%)\end{array}$ & 1 (Ref.) & 1 (Ref.) \\
\hline $\begin{array}{l}\text { MedDiets } \\
\text { combined }\end{array}$ & $\begin{array}{c}19 / 2098 \\
(0.91 \%)\end{array}$ & $\begin{array}{c}0.34 \\
{[0.18 ; 0.64]}\end{array}$ & $\begin{array}{c}0.44 \\
{[0.23 ; 0.83]}\end{array}$ & $\begin{array}{l}40 / 2098 \\
(1.91 \%)\end{array}$ & $\begin{array}{c}0.96 \\
{[0.55 ; 1.69]}\end{array}$ & $\begin{array}{c}1.29 \\
{[0.74 ; 2.26]}\end{array}$ \\
\hline MedDiet-EVOO & $\begin{array}{c}10 / 1128 \\
(0.89 \%)\end{array}$ & $\begin{array}{c}0.29 \\
{[0.14 ; 0.62]}\end{array}$ & $\begin{array}{c}0.36 \\
{[0.16 ; 0.80]}\end{array}$ & $\begin{array}{c}24 / 1128 \\
(2.13 \%)\end{array}$ & $\begin{array}{c}1.04 \\
{[0.56 ; 1.93]}\end{array}$ & $\begin{array}{c}1.57 \\
{[0.84 ; 2.97]}\end{array}$ \\
\hline MedDiet-Nuts & $\begin{array}{c}9 / 970 \\
(0.93 \%)\end{array}$ & $\begin{array}{c}0.40 \\
{[0.18 ; 0.89]}\end{array}$ & $\begin{array}{c}0.56 \\
{[0.26 ; 1.21]}\end{array}$ & $\begin{array}{l}16 / 970 \\
(1.65 \%)\end{array}$ & $\begin{array}{c}0.86 \\
{[0.44 ; 1.69]}\end{array}$ & $\begin{array}{c}1.04 \\
{[0.52 ; 2.06]}\end{array}$ \\
\hline
\end{tabular}

${ }^{1}$ Thrombocytopenia has been defined as the presence of $<122 \times 10^{9}$ platelets/L in men and $<140 \times 10^{9}$ platelets/L in women, and thrombocytosis has been defined as the presence of $>350 \times 10^{9}$ platelets $/ \mathrm{L}$ in men and $>379 \times 10^{9}$ platelets $/ \mathrm{L}$ in women. Hazard ratios were estimated by multivariable Cox proportional hazards regression models. Model 1 was stratified by sex and recruitment site and adjusted for age. Model 2 was further stratified by educational level and adjusted for baseline platelet count, diabetes, hypercholesterolemia, hypertriglyceridemia, hypertension, antiplatelet drug use, smoking habit, white blood cells, leisure-time physical activity, body mass index, intake of alcohol, folates, and iron (at baseline, all), as well as two propensity scores that used 30 baseline variables to estimate the probability of assignment to each of the intervention groups. We used robust standard errors to account for intra-cluster correlations. HR: hazard ratio; MedDiet-EVOO: Mediterranean diet intervention enriched with extra-virgin olive oil; MedDiet-Nuts: Mediterranean diet intervention enriched with mixed nuts.

\subsection{Interaction between Platelet Count-Related Disorders at Baseline and MedDiet on All-Cause Mortality}

Thrombocytopenia was robustly associated with a higher risk of all-cause mortality (HR: $4.71[2.69 ; 8.24])$. This relationship was stronger for participants in the control diet group and blunted in those allocated to the MedDiet intervention groups $\left(\mathrm{HR}_{\text {control diet }}\right.$ : 10.9 [5.26; 22.8]; HR MedDiet: 2.18 [0.95; 5.00]; $p$-interaction: 0.018) (Figure 2A). No interaction between thrombocytosis at baseline and group allocation on all-cause mortality was found (Figure 2B). Specific values are available in Supplementary Materials Table S2. 
A. Thrombocytopenia

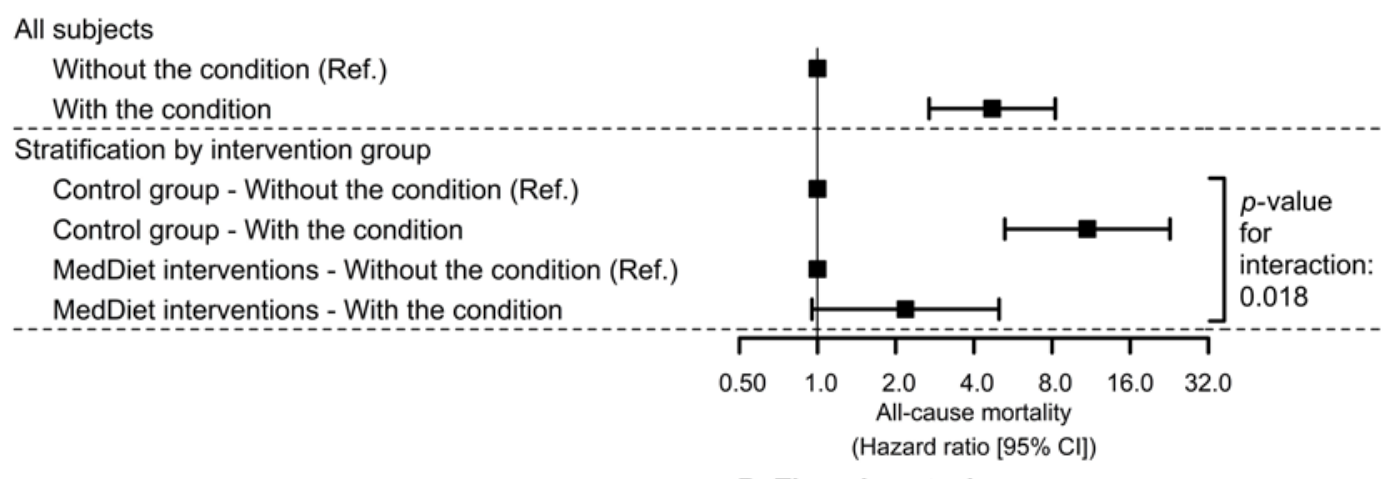

B. Thrombocytosis

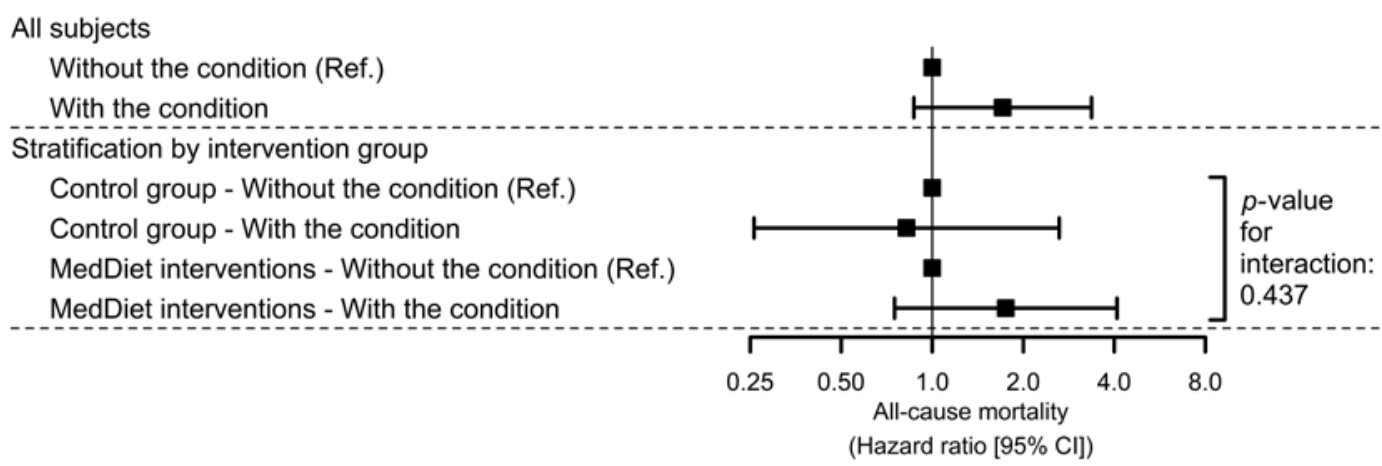

Figure 2. Associations of thrombocytopenia (A) and thrombocytosis (B) at baseline with all-cause mortality risk stratified by MedDiet groups. Hazard ratios were estimated by multivariable Cox proportional hazards regression models stratified by sex, recruitment site, and educational level and adjusted for age, baseline platelet count, diabetes, hypercholesterolemia, hypertriglyceridemia, hypertension, antiplatelet drug use, smoking habit, white blood cells, leisure-time physical activity, body mass index, intake of alcohol, folates, and iron (at baseline, all), as well as two propensity scores that used 30 baseline variables to estimate the probability of assignment to each of the intervention groups. We used robust standard errors to account for intra-cluster correlations. MedDiet: Mediterranean diet.

\section{Discussion}

Our findings suggest that MedDiet contributed to maintaining platelet counts within the normal range in an older population at high cardiovascular risk. In addition, MedDiet interventions also decreased the risk of developing thrombocytopenia and attenuated the association of this platelet count-related alteration with all-cause mortality.

Low and high platelet counts are risk factors for increased mortality and cardiovascular incidence in the general population and high cardiovascular risk individuals [9-11]. In the particular case of high platelet count, it has been associated with greater cardiovascular disease and cancer incidence and mortality $[10,26]$. The association of platelet count with cardiovascular disease and some types of cancer (lung) has been proven to be causal in some Mendelian randomization studies $[27,28]$. Therefore, reducing platelets via lifestyle intervention could have high therapeutic value. Our findings suggest that platelet count tends to increase over time and show that following a MedDiet contributes to maintaining platelet counts within normal levels in an older population at high cardiovascular risk. This last effect was particularly marked in individuals starting off with near-high platelet count values, who are likely to benefit from a decrease [26]. In view of our findings, we could expect that platelet counts in MedDiet groups could be $16 \cdot 10^{9}$ counts/L lower when compared to the control diet at the end of the study follow-up, although this decrease seemed insufficient to decrease the risk of developing thrombocytosis. An increase in the genetically predetermined levels of platelets of $51.1 \cdot 10^{9}$ platelets $/ \mathrm{L}$ in men and $55.3 \cdot 10^{9}$ platelets $/ \mathrm{L}$ in women was associated with a $7 \%$ increase in the risk of ischemic stroke and a $12 \%$ increment in risk of cardioembolic stroke independently from the baseline platelet count [27]. 
Assuming a linear, continuous association between platelet levels and outcome risk, a reduction in platelet count similar to the one observed in our study would be linked to a 2.0-2.2\% decrease in ischemic stroke risk and a 3.5-3.8\% decline in cardioembolic stroke risk, a modest reduction in the risk of cerebrovascular event but within the magnitude expected for a realistic lifestyle modification. An association between high adherence to a MedDiet and reduced platelet count was reported in a cross-sectional study [13] and in a small-scale, short-term intervention trial [14], and another plant-based dietary pattern (vegan diet) was shown to be able to moderate this parameter in the short term in a recent small-sized study [12]. However, this is the first time that a long-term effect has been reported in a large randomized controlled trial with repeated measurements. The capacity of the MedDiet to decrease inflammation could help with explaining this effect $[29,30]$, given the close relationship between inflammatory status and platelet levels [31].

Low platelet count states are associated with a greater risk of infectious disease and mortality in cohort studies on the general population [9], in individuals at high cardiovascular risk [32], and in acute conditions, such as COVID-19 infection [33]. Beyond the maintenance of platelet count within normal ranges, MedDiet interventions appeared to lower the risk of developing thrombocytopenia in older adults at high cardiovascular risk, and attenuated all-cause mortality risk among individuals presenting the condition. Deficient levels of platelets and their consequences are traditionally addressed by pharmacological treatments [34]. However, to the best of our knowledge, this is the first intervention study to date to describe the protective effect of a healthy diet on the development of low platelet count and their associated mortality. Our main hypothesis to explain the decrease in the risk of developing thrombocytopenia and some of its potential complications was the improvement in the general nutritional status of the participants in the MedDiet intervention groups, which may subsequently lead to an improved production of platelets in those participants at risk of presenting low platelet count, since platelet production and thrombocytopenia risk appear to be highly dependent on the nutritional status of the individuals [15].

Our study presents some limitations. First, platelet count alterations were not a predefined endpoint in the PREDIMED study and, therefore, our findings should be considered as exploratory. Second, our participants were investigated between 2003 and 2009, and thus it would be advisable that our findings should be re-evaluated in more recent studies. Third, we cannot generalize our findings to other populations than older adults with a high prevalence of cardiovascular risk factors. Fourth, platelet count alterations were defined according to threshold values obtained in Mediterranean populations and had a low incidence in our study. Therefore, our results should be interpreted with caution. Fifth, platelet levels can present short-term fluctuations, but we overcame this limitation by using repeated measures. Sixth, considering that MedDiet interventions were modest real-life dietary modifications and that the control diet was a healthy dietary pattern, only moderate effects on platelet-related outcomes could be reported. Finally, residual confounding may have resulted from self-reported information on leisure-time physical activity and the consumption of alcohol, dietary folate, and iron.

\section{Conclusions}

In conclusion, following a MedDiet intervention contributed to maintaining platelet counts within normal ranges, a surrogate risk factor for several chronic diseases that tended to increase over time. This effect was particularly strong in participants presenting nearhigh platelet counts at baseline. In parallel, the MedDiet intervention also decreased the risk of developing thrombocytopenia and attenuated the association of this platelet count alteration with all-cause mortality. Despite the limitation of the small number of subjects in some subgroups, to the best of our knowledge, this is the first intervention study reporting beneficial effects of a healthy dietary pattern on thrombocytopenia and its related disorders. In parallel, our findings also confirm the long-term capacity of MedDiet to moderate the increase in platelet count over time. Our results suggest the capacity of MedDiet to keep 
platelet count in its typical range, which may have a great clinical impact, since both low and high platelet counts are risk factors for several lethal outcomes.

Supplementary Materials: The following are available online at https://www.mdpi.com/2072-6 643/13/2/559/s1: Figure S1: Weighted Kaplan-Meier estimates of the cumulative incidence of thrombocytopenia and thrombocytosis in intervention groups; Table S1: Power analyses; Table S2: Associations of platelet count alterations at baseline with the risk of all-cause mortality stratified by Mediterranean diet intervention group.

Author Contributions: Conceptualization: Á.H.; methodology: Á.H., C.L., and S.C.-B.; formal analysis; Á.H.; data curation: E.R., A.T.-R., O.C., X.P., Z.V.-R., J.V.S., J.S.-S., J.L., E.G.-G., Á.M.A.-G., M.F., L.S.-M., E.S., C.R., D.C., M.G.-F., M.C., and R.E.; writing-original draft preparation: Á.H., C.L., and S.C.-B.; writing—review and editing: E.R., A.T.-R., O.C., X.P., Z.V.-R., J.V.S., J.S.-S., J.L., E.G.-G., Á.M.A.-G., M.F., L.S.-M., E.S., C.R., D.C., M.G.-F., M.C., and R.E.; visualization: Á.H.; supervision: E.R., J.S.-S., J.L., E.G.-G., Á.M.A.-G., M.F., L.S.-M., D.C., and R.E.; project administration: Á.H., A.T.-R., O.C., X.P., and R.E.; funding acquisition: A.T.-R., O.C., X.P., and R.E. All authors have read and agreed to the published version of the manuscript.

Funding: This work was supported by grants from Instituto de Salud Carlos III (grant numbers CB06/03/0019, CB06/03/0028, CD17/00122, PIE14/00045_INFLAMES, and OBN17PI02), Agència de Gestió d'Ajuts Universitaris i de Recerca (grant numbers 2017 SGR 222 and 2017-BP-00021), and the Spanish Ministry of Science, Innovation, and Universities (grant number FPU17/00785).

Institutional Review Board Statement: The study was conducted according to the guidelines of the Declaration of Helsinki and approved by the Institutional Ethics Committee of PSMAR (protocol code: 2018/8180/I, date of approval: 4 December 2018).

Informed Consent Statement: Informed consent was obtained from all subjects involved in the study. The information and informed content sheets are available among the Supplementary Materials.

Data Availability Statement: The dataset analyzed during the current study is not publicly available due to national data regulations and for ethical reasons, including that we do not have the explicit written consent of the study volunteers to make their deidentified data available at the end of the study. However, datasets and R codes of data management/transformation and statistical analyses can be requested by sending a letter to the PREDIMED Steering Committee (predimed-steeringcommitte@googlegroups.com). The request will then be passed to all the members of the committee for deliberation.

Acknowledgments: A full list of names of all study collaborators is available in the Supplementary Appendix. J.S.-S. gratefully acknowledges the financial support by ICREA under the ICREA Academia program. CIBER de Fisiopatología de la Obesidad y Nutrición is an initiative of the Instituto de Salud Carlos III, Madrid, Spain, and financed by the European Regional Development Fund.

Conflicts of Interest: E.R. reports personal fees, grants, and nonfinancial support from the California Walnut Commission and Alexion; personal fees and nonfinancial support from Danone; and nonfinancial support from the International Nut Council. X.P. reports being a board member, lecture fees, and grants from Ferrer International; being a board member and grants from the Residual Risk Reduction Initiative Foundation; personal fees from Abbott Laboratories; lecture fees and grants from Merck and Roche; lecture fees from Danone, Esteve, Menarini, Mylan, LACER, and Rubio Laboratories; and grants from Sanofi, Kowa, Unilever, Boehringer Ingelheim, and Karo Bio. J.S.-S. reports being a board member and personal fees from Instituto Danone Spain; being a board member and grants from the International Nut and Dried Fruit Foundation; personal fees from Aguas Font Vella Lanjarón, and Danone S.A; and grants from Eroski Distributors. L.S.-M. reports being a board member of the Mediterranean Diet Foundation and the Beer and Health Foundation. R.E. reports being a board member of the Research Foundation on Wine and Nutrition, the Beer and Health Foundation, and the European Foundation for Alcohol Research; personal fees from KAO Corporation; lecture fees from Instituto Cerventes, Fundacion Dieta Mediterranea, Cerveceros de España, Lilly Laboratories, AstraZeneca, and Sanofi; and grants from Novartis, Amgen, Bicentury, and Grand Fountaine. The rest of the authors have nothing to disclose. The funders had no role in the design of the study; in the collection, analyses, or interpretation of data; in the writing of the manuscript, or in the decision to publish the results. 


\section{References}

1. Estruch, R.; Ros, E.; Salas-Salvadó, J.; Covas, M.-I.; Corella, D.; Arós, F.; Gómez-Gracia, E.; Ruiz-Gutiérrez, V.; Fiol, M.; Lapetra, J.; et al. Primary Prevention of Cardiovascular Disease with a Mediterranean Diet Supplemented with Extra-Virgin Olive Oil or Nuts. N. Engl. J. Med. 2018, 378, e34. [CrossRef]

2. Martínez-González, M.A.; Gea, A.; Ruiz-Canela, M. The Mediterranean Diet and Cardiovascular Health. Circ. Res. 2019, 124, 779-798. [CrossRef] [PubMed]

3. Martínez-González, M.A.; Salas-Salvadó, J.; Estruch, R.; Corella, D.; Fitó, M.; Ros, E.; PREDIMED INVESTIGATORS. Benefits of the Mediterranean Diet: Insights From the PREDIMED Study. Prog. Cardiovasc. Dis. 2015, 58, 50-60. [CrossRef] [PubMed]

4. Hernáez, Á.; Castañer, O.; Tresserra-Rimbau, A.; Pintó, X.; Fitó, M.; Casas, R.; Martínez-González, M.Á.; Corella, D.; Salas-Salvadó, J.; Lapetra, J.; et al. Mediterranean Diet and Atherothrombosis Biomarkers: A Randomized Controlled Trial. Mol. Nutr. Food Res. 2020, 64, e2000350. [CrossRef] [PubMed]

5. Pignatelli, P.; Pastori, D.; Farcomeni, A.; Nocella, C.; Bartimoccia, S.; Vicario, T.; Bucci, T.; Carnevale, R.; Violi, F. Mediterranean diet reduces thromboxane A2 production in atrial fibrillation patients. Clin. Nutr. 2015, 34, 899-903. [CrossRef]

6. Chiva-Blanch, G.; Sala-Vila, A.; Crespo, J.; Ros, E.; Estruch, R.; Badimon, L. The Mediterranean diet decreases prothrombotic microvesicle release in asymptomatic individuals at high cardiovascular risk. Clin. Nutr. 2020, 39, 3377-3384. [CrossRef]

7. Zucoloto, A.Z.; Jenne, C.N. Platelet-Neutrophil Interplay: Insights Into Neutrophil Extracellular Trap (NET)-Driven Coagulation in Infection. Front. Cardiovasc. Med. 2019, 6, 85. [CrossRef]

8. Han, S.; Wu, P.; Duan, M.; Yang, F.; He, W.; Wu, N.; Hu, X.; Gan, D.; Wang, G.; Yang, M.; et al. The crosstalk between platelets and body fat: A reverse translational study. Clin. Nutr. 2020. [CrossRef]

9. Bonaccio, M.; Di Castelnuovo, A.; Costanzo, S.; De Curtis, A.; Donati, M.B.; Cerletti, C.; De Gaetano, G.; Iacoviello, L. Age- and sex-based ranges of platelet count and cause-specific mortality risk in an adult general population: Prospective findings from the Moli-sani study. Platelets 2018, 29, 312-315. [CrossRef]

10. Song, P.S.; Ahn, K.T.; Jeong, J.-O.; Jeon, K.-H.; Song, Y.B.; Gwon, H.-C.; Rha, S.-W.; Jeong, M.H.; Seong, I.-W. Association of baseline platelet count with all-cause mortality after acute myocardial infarction. Eur. Heart J. Acute Cardiovasc. Care 2020. [CrossRef]

11. Patti, G.; Di Martino, G.; Ricci, F.; Renda, G.; Gallina, S.; Hamrefors, V.; Melander, O.; Sutton, R.; Engström, G.; De Caterina, R.; et al. Platelet Indices and Risk of Death and Cardiovascular Events: Results from a Large Population-Based Cohort Study. Thromb. Haemost. 2019, 119, 1773-1784. [CrossRef]

12. Lederer, A.K.; Maul-Pavicic, A.; Hannibal, L.; Hettich, M.; Steinborn, C.; Gründemann, C.; Zimmermann-Klemd, A.M.; Müller, A.; Sehnert, B.; Salzer, U.; et al. Vegan diet reduces neutrophils, monocytes and platelets related to branched-chain amino acids-A randomized, controlled trial. Clin. Nutr. 2020, 39, 3241-3250. [CrossRef]

13. Bonaccio, M.; Di Castelnuovo, A.; De Curtis, A.; Costanzo, S.; Persichillo, M.; Donati, M.B.; Cerletti, C.; Iacoviello, L.; De Gaetano, G.; Moli-sani Project Investigators. Adherence to the Mediterranean diet is associated with lower platelet and leukocyte counts: Results from the Moli-sani study. Blood 2014, 123, 3037-3044. [CrossRef]

14. Ambring, A.; Johansson, M.; Axelsen, M.; Gan, L.M.; Strandvik, B.; Friberg, P. Mediterranean-inspired diet lowers the ratio of serum phospholipid n-6 to n-3 fatty acids, the number of leukocytes and platelets, and vascular endothelial growth factor in healthy subjects. Am. J. Clin. Nutr. 2006, 83, 575-581. [CrossRef]

15. Swain, F.; Bird, R. How I approach new onset thrombocytopenia. Platelets 2020, 31, 285-290. [CrossRef]

16. Soltani, S.; Jayedi, A.; Shab-Bidar, S.; Becerra-Tomás, N.; Salas-Salvadó, J. Adherence to the Mediterranean Diet in Relation to All-Cause Mortality: A Systematic Review and Dose-Response Meta-Analysis of Prospective Cohort Studies. Adv. Nutr. 2019, 10, 1029-1039. [CrossRef]

17. Martinez-Gonzalez, M.A.; Corella, D.; Salas-Salvado, J.; Ros, E.; Covas, M.I.; Fiol, M.; Warnberg, J.; Aros, F.; Ruiz-Gutierrez, V.; Lamuela-Raventos, R.M.; et al. Cohort Profile: Design and methods of the PREDIMED study. Int. J. Epidemiol. 2012, 41, 377-385. [CrossRef] [PubMed]

18. Danese, E.; Montagnana, M.; Favaloro, E.J.; Lippi, G. Drug-Induced Thrombocytopenia: Mechanisms and Laboratory Diagnostics. Semin. Thromb. Hemost. 2020, 46, 264-274. [CrossRef]

19. Babio, N.; Ibarrola-Jurado, N.; Bulló, M.; Martínez-González, M.Á.; Wärnberg, J.; Salaverría, I.; Ortega-Calvo, M.; Estruch, R.; Serra-Majem, L.; Covas, M.I.; et al. White Blood Cell Counts as Risk Markers of Developing Metabolic Syndrome and Its Components in the Predimed Study. PLoS ONE 2013, 8, e58354. [CrossRef] [PubMed]

20. Biino, G.; Santimone, I.; Minelli, C.; Sorice, R.; Frongia, B.; Traglia, M.; Ulivi, S.; Di Castelnuovo, A.; Gögele, M.; Nutile, T.; et al. Age- And Sex-Related Variations in Platelet Count in Italy: A Proposal of Reference Ranges Based on 40987 Subjects' Data. PLoS ONE 2013, 8, e54289. [CrossRef] [PubMed]

21. Weiliang Qiu, A.; Chavarro, J.; Weiliang Qiu, M.; Qiu, W.; Chavarro, J.; Lazarus, R.; Rosner, B.; Ma, J. Package "PowerSurvEpi": Power and Sample Size Calculation for Survival Analysis of Epidemiological Studies. 2018. Available online: https://cran.rproject.org/web/packages/powerSurvEpi/powerSurvEpi.pdf (accessed on 8 January 2021).

22. Bates, D.; Mächler, M.; Bolker, B.; Walker, S. Fitting Linear Mixed-Effects Models Using lme4. J. Stat. Softw. 2015, 67, 1-48. [CrossRef]

23. Stringhini, S.; Zaninotto, P.; Kumari, M.; Kivimäki, M.; Batty, G.D. Lifecourse socioeconomic status and type 2 diabetes: The role of chronic inflammation in the English Longitudinal Study of Ageing. Sci. Rep. 2016, 6, 24780. [CrossRef] 
24. Therneau, T.M. Package "Survival": Survival Analysis. 2018. Available online: https://cran.r-project.org/web/packages/ survival/survival.pdf (accessed on 21 December 2020).

25. R Core Team. R: A Language and Environment for Statistical Computing; R Foundation for Statistical Computing: Vienna, Austria, 2014; Available online: https:/ / www.r-project.org/ (accessed on 21 December 2020).

26. Vinholt, P.J.; Hvas, A.M.; Frederiksen, H.; Bathum, L.; Jørgensen, M.K.; Nybo, M. Platelet count is associated with cardiovascular disease, cancer and mortality: A population-based cohort study. Thromb. Res. 2016, 148, 136-142. [CrossRef]

27. Gill, D.; Monori, G.; Georgakis, M.K.; Tzoulaki, I.; Laffan, M. Genetically Determined Platelet Count and Risk of Cardiovascular Disease: Mendelian Randomization Study. Arterioscler. Thromb. Vasc. Biol. 2018, 38, 2862-2869. [CrossRef] [PubMed]

28. Zhu, Y.; Wei, Y.; Zhang, R.; Dong, X.; Shen, S.; Zhao, Y.; Bai, J.; Albanes, D.; Caporaso, N.E.; Landi, M.T.; et al. Elevated platelet count appears to be causally associated with increased risk of lung cancer: A mendelian randomization analysis. Cancer Epidemiol. Biomarkers Prev. 2019, 28, 935-942. [CrossRef] [PubMed]

29. Casas, R.; Sacanella, E.; Urpí-Sardà, M.; Chiva-Blanch, G.; Ros, E.; Martínez-González, M.-A.; Covas, M.-I.; Salas-Salvadó, J.; Fiol, M.; Arós, F.; et al. The Effects of the Mediterranean Diet on Biomarkers of Vascular Wall Inflammation and Plaque Vulnerability in Subjects with High Risk for Cardiovascular Disease. A Randomized Trial. PLoS ONE 2014, 9, e100084. [CrossRef]

30. Casas, R.; Sacanella, E.; Urpí-Sardà, M.; Corella, D.; Castañer, O.; Lamuela-Raventos, R.-M.; Salas-Salvadó, J.; MartínezGonzález, M.-A.; Ros, E.; Estruch, R. Long-Term Immunomodulatory Effects of a Mediterranean Diet in Adults at High Risk of Cardiovascular Disease in the PREvención con DIeta MEDiterránea (PREDIMED) Randomized Controlled Trial. J. Nutr. 2016, 146, 1684-1693. [CrossRef] [PubMed]

31. Vogel, S.; Thein, S.L. Platelets at the crossroads of thrombosis, inflammation and haemolysis. Br. J. Haematol. 2018, 180, 761-767. [CrossRef]

32. Goliasch, G.; Forster, S.; El-Hamid, F.; Sulzgruber, P.; Meyer, N.; Siostrzonek, P.; Maurer, G.; Niessner, A. Platelet count predicts cardiovascular mortality in very elderly patients with myocardial infarction. Eur. J. Clin. Investig. 2013, 43, 332-340. [CrossRef]

33. Lippi, G.; Plebani, M.; Henry, B.M. Thrombocytopenia is associated with severe coronavirus disease 2019 (COVID-19) infections: A meta-analysis. Clin. Chim. Acta 2020, 506, 145-148. [CrossRef]

34. Puavilai, T.; Thadanipon, K.; Rattanasiri, S.; Ingsathit, A.; McEvoy, M.; Attia, J.; Thakkinstian, A. Treatment efficacy for adult persistent immune thrombocytopenia: A systematic review and network meta-analysis. Br. J. Haematol. 2020, 188, 450-459. [CrossRef] [PubMed] 